TRANSACTIONS OF THE

AMERICAN MATHEMATICAL SOCIETY

Volume 351, Number 7, Pages 2763-2775

S 0002-9947(99)02376-4

Article electronically published on February 15, 1999

\title{
AN EQUIVARIANT SMASH SPECTRAL SEQUENCE AND AN UNSTABLE BOX PRODUCT
}

\author{
MICHELE INTERMONT
}

\begin{abstract}
Let $G$ be a finite group. We construct a first quadrant spectral sequence which converges to the equivariant homotopy groups of the smash product $X \wedge Y$ for suitably connected, based $G$-CW complexes $X$ and $Y$. The $E^{2}$ term is described in terms of a tensor product functor of equivariant $\Pi$ algebras. A homotopy version of the non-equivariant Künneth theorem and the equivariant suspension theorem of Lewis are both shown to be special cases of the corner of the spectral sequence.

We also give a categorical description of this tensor product functor which is analogous to the description in equivariant stable homotopy theory of the box product of Mackey functors. For this reason, the tensor product functor deserves to be called an "unstable box product".
\end{abstract}

\section{INTRODUCTION}

In [19] it was shown that there is a spectral sequence converging to the nonequivariant homotopy groups of the wedge of two based, connected CW complexes, and another spectral sequence converging to the homotopy groups of the smash product of two such complexes. In each, the $E^{2}$ term depends only on the homotopy groups and the primary homotopy operations of each of the two spaces. The spectral sequence for a wedge was extended in [9] to a spectral sequence converging to the equivariant homotopy groups of the wedge of two suitably connected, based $G$-CW complexes when $G$ is a finite group.

The significance of the spectral sequence in [19] which converges to the smash product is that the $E^{2}$ term can be described using a tensor product functor (and its derived functors). In this paper we extend the smash spectral sequence result to the equivariant setting. We also show that the tensor product functor deserves to be called an "unstable box product" because of its relation to the box product found in equivariant stable homotopy theory. Indeed, the categorical description of the tensor product in Theorem 4.6 is analogous to that of the box product given by Lewis in [11].

Let $V$, with dimension $|V|$, be a representation of a group $G$. The representation $V \oplus R^{m}, m \geq 0$, will be denoted $(V+m)$. Here $G$ acts trivially on the Euclidean space. We say a $G$-space $X$ is $V^{*}$-connected if, for each subgroup $H$ of $G$, the fixed

Received by the editors August 18, 1997.

1991 Mathematics Subject Classification. Primary 55P91, 55Q91, 55T99, 18G10; Secondary 55P40, 55Q35, 55U25, 18G15, 55U10.

Key words and phrases. Smash product, box product, tensor product functor, equivariant spectral sequence, Kan extension, Künneth theorem, Freudenthal suspension theorem, equivariant homotopy theory. 
point set $X^{H}$ is $V^{H}$-connected. The $\Pi(V)$-algebra determined by $X$, written $\Pi_{V} X$, is the collection $\left\{\pi_{V+m}^{H}(X)\right\}_{m, H}, m \geq 0, H \subseteq G$ together with all the homotopy operations between these groups (set, if $m=0$ ).

Throughout this paper, $V$ and $W$ will be fixed, finite dimensional representations of the fixed, finite group $G$. The first main result is:

Theorem 1.1. Let $X$ and $Y$ be based $G$-CW complexes such that $X$ is $V^{*}$-connected and $Y$ is $W^{*}$-connected. For each $H \subseteq G$ there is a first quadrant spectral sequence $\left\{E_{p, q}^{r}(H)\right\}$ converging to $\left\{\pi_{V+W+p+q}^{H}(X \wedge Y)\right\}_{p+q}$, where $p, q, p+q \geq 0$. For fixed $p$ the collection of columns $E_{p, *}^{2}:=\left\{E_{p, *}^{2}(H)\right\}_{H}$ is isomorphic to the $p^{\text {th }}$ derived functor of the tensor product functor. That is, $E_{p, *}^{2} \cong \Pi_{V} X \otimes_{p} \Pi_{W} Y$.

A precise formulation of the next theorem will be given in Theorem 4.6, but for now we state:

Theorem. Let $A$ be $a \Pi(V)$-algebra, $B$ a $\Pi(W)$-algebra. The tensor product functor of $A$ and $B$ and the unstable box product functor of $A$ and $B$ are equivalent.

In the corner of the spectral sequence, we see the expected familiar results. Corollary 4.7 is the homotopy version of the non-equivariant Künneth theorem, and Corollary 4 is the Freudenthal suspension theorem of Lewis ([12, Theorem $2.5])$.

The organization of this paper is as follows. Section 2 fixes some notation and establishes the spectral sequences of Theorem 1.1. In Section 3, $\Pi(V)$-algebras are reviewed and the proof of Theorem 1.1 is finished. Finally, the second main theorem and the corollaries are presented in Section 4.

The author wishes to thank L. Gaunce Lewis, Jr. for his many generous remarks, and William G. Dwyer and James M. Turner for several helpful conversations.

\section{The spectral Sequence}

Recall that for a fixed representation $V, S^{V}$ is the one-point compactification of $V$ with the compactification point taken as the basepoint. This point is fixed by the action of $G$. Since $V$ is a representation, the set of $H$ fixed points, $\left(S^{V}\right)^{H}$, is again a sphere for each subgroup $H$ of $G$. If $X$ is a $G$-space, then $\pi_{V}^{H}(X)$ is defined to be $\left[S^{V}, X\right]_{H}$, the based $H$-homotopy classes of based $H$-maps. This is a group as long as the dimension $\left|V^{H}\right|$ of $V^{H}$ is greater than or equal to 1 , and an abelian group if $\left|V^{H}\right| \geq 2$. Notice that $\left[S^{V}, X\right]_{H} \cong\left[S^{V} \wedge G / H_{+}, X\right]_{G}$. The object $S^{V} \wedge G / H_{+}$will be abbreviated as $S_{H}^{V}$, and the corresponding equivariant disk $D^{V+1} \wedge G / H_{+}$will be abbreviated as $D_{H}^{V+1}$. Note too, that if $G$ acts trivially on $V$, then $\pi_{V}^{H}(X)=\pi_{m}\left(X^{H}\right)$ where $m=|V|$. If $X$ and $Y$ are $G$-spaces, then the space $M \operatorname{pp}_{*}(X, Y)$ of pointed maps between them is a $G$-space under the conjugation action of $G$. The fixed points are the pointed $G$-maps between $X$ and $Y$, written $\operatorname{Map}_{*}^{G}(X, Y)$. The space $X \wedge Y$ derives its $G$ action from the diagonal action on $X \times Y$ as the cofibre of the inclusion $G$-map $X \vee Y \hookrightarrow X \times Y$.

Let $X$ be a based, $V^{*}$-connected $G$-CW complex. In [9], following [19], we constructed a simplicial $G$-space $X_{\bullet}$ such that each $X_{i}$ is $G$-homotopy equivalent to a wedge of $G$-spheres $S_{H_{i}}^{V+m_{i}}, m_{i} \geq 1$, and such that $\left|X_{\bullet}\right| \simeq_{G} X$. We recall this functorial construction of $X_{\bullet}$. The space $\mathcal{V} X$ is given by the pushout 


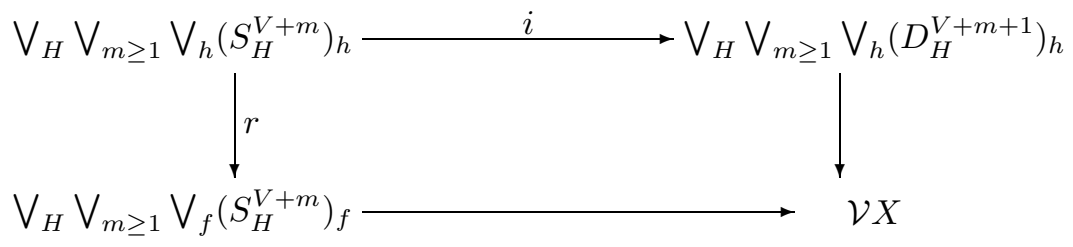

indexed over $h \in \operatorname{Map}_{*}^{G}\left(D_{H}^{V+m+1}, X\right), f \in \operatorname{Map}_{*}^{G}\left(S_{H}^{V+m}, X\right)$. The $G$-map $i$ is the inclusion of the boundary of a disk into the disk itself, and the $G$-map $r$ is the map which takes each wedge summand $\left(S_{H}^{V+m}\right)_{h}$ to the wedge summand $\left(S_{H}^{V+m}\right)_{f}$ where $f: S_{H}^{V+m} \rightarrow X$ is the restriction of $h$ to the boundary of $D_{H}^{V+m+1}$. $G$ acts on $\mathcal{V} X$ in a natural way.

There are two natural maps with $\mathcal{V} X$ :

(i) $\epsilon: \mathcal{V} X \rightarrow X$ which sends $\left(S_{H}^{V+m}\right)_{f}$ into $X$ by the indexing map $f$ and $\left(D_{H}^{V+m+1}\right)_{h}$ into $X$ by the indexing map $h$.

(ii) $\beta: \mathcal{V} X \rightarrow \mathcal{V}^{2} X$ which sends $\left(S_{H}^{V+m}\right)_{f} G$-homeomorphically to the copy of $S_{H}^{V+m}$ in $\mathcal{V}^{2} X$ indexed by the inclusion of the sphere into $\mathcal{V} X$ and which sends $\left(D_{H}^{V+m+1}\right)_{h} G$-homeomorphically to the copy of $D_{H}^{V+m+1}$ in $\mathcal{V}^{2} X$ indexed by the inclusion.

The construction $\mathcal{V}$ together with the maps $\epsilon, \beta$ form a cotriple (or comonad, in the language of [15]) from which a cellular simplicial $G$-space can be constructed ([9], following [8]). We call this simplicial $G$-space the generalized $G(V)$-sphere resolution of $X$, and denote it by $X_{\bullet}$. The spaces $X_{i}$ are defined as

$$
X_{i}:=\mathcal{V}^{i+1} X=\mathcal{V}\left(\mathcal{V}^{i} X\right), \quad i \geq 0
$$

and $X_{\bullet}$ is augmented by $\epsilon: X_{0} \rightarrow X$. The face and degeneracy maps, $0 \leq j \leq p$, are given by

$$
d_{j}=\mathcal{V}^{j} \epsilon \mathcal{V}^{p-j}: X_{p} \rightarrow X_{p-1} \text { and } s_{j}=\mathcal{V}^{j} \beta \mathcal{V}^{p-j}: X_{p} \rightarrow X_{p+1} .
$$

We record the promised equivalence:

Proposition 2.1 ([9, Corollary 4.5]). Let $X$ be a based $G-C W$ complex, $X$. as above. If $X$ is $V^{*}$-connected, then the augmentation map, $\epsilon: X_{0} \rightarrow X$, induces a G-homotopy equivalence $\left|X_{\bullet}\right| \rightarrow X$.

Lemma 2.2. Let $V$ and $W$ be $G$-representations. Let $X, Y$ be based $G$-CW complexes with $X_{\bullet}, Y_{\bullet}$ the $G(V)$ - and $G(W)$-sphere resolutions of $X$ and $Y$ respectively. If $X$ is $V^{*}$-connected and $Y$ is $W^{*}$-connected, then $\left|X \bullet \wedge Y_{\bullet}\right|$ is $G$-homotopy equivalent to $X \wedge Y$.

Proof. By Proposition 2.1, there are homotopy equivalences $\left|X_{\bullet}\right|^{H} \stackrel{\simeq}{\rightarrow} X^{H}$ and $\left|Y_{\bullet}\right|^{H} \stackrel{\cong}{\rightarrow} Y^{H}$ for all $H \subseteq G$. Thus, for all $H \subseteq G,\left|X_{\bullet}\right|^{H} \vee\left|Y_{\bullet}\right|^{H} \cong X^{H} \vee Y^{H}$, and $\left|X_{\bullet}\right|^{H} \times\left|Y_{\bullet}\right|^{H} \cong X^{H} \times Y^{H}$. Taking fixed points is compatible with the action on $X \vee Y$ and with the diagonal action on the product, so we have

$$
\left(\left|X_{\bullet}\right| \vee\left|Y_{\bullet}\right|\right)^{H} \cong(X \vee Y)^{H} \text { and }\left(\left|X_{\bullet}\right| \times\left|Y_{\bullet}\right|\right)^{H} \cong(X \times Y)^{H}
$$

Now $\left(\left|X_{\bullet}\right| \times\left|Y_{\bullet}\right|\right)^{H} \cong\left|X_{\bullet} \times Y_{\bullet}\right|^{H}$, and likewise, this holds for the wedge, so the cofibres of $\left|X_{\bullet} \vee Y_{\bullet}\right|^{H} \hookrightarrow\left|X_{\bullet} \times Y_{\bullet}\right|^{H}$ and $(X \vee Y)^{H} \hookrightarrow(X \times Y)^{H}$ are homotopy equivalent for all $H \subseteq G$. That is, $\left|X_{\bullet} \wedge Y_{\bullet}\right|^{H} \simeq(X \wedge Y)^{H}$. This is enough to conclude that $\left|X_{\bullet} \wedge Y_{\bullet}\right|$ and $X \wedge Y$ are $G$-homotopy equivalent ([6, II $\left.\S 2.7\right]$ ). 
Notice that $X_{\bullet} \wedge Y_{\bullet}$ is $(V+W+1)^{*}$-connected, and thus, $G$-homotopy equivalence does indeed imply that $\pi_{V+W+*}\left|X_{\bullet} \wedge Y_{\bullet}\right| \cong \pi_{V+W+*} X \wedge Y$. We now have

Theorem 2.3. Let $X$ be a based, $V^{*}$-connected $G$-CW complex, and $Y$ a based, $W^{*}$-connected $G$ - $C W$ complex with $X_{\bullet}, Y_{\bullet}$ their respective $G(V)$ - and $G(W)$-sphere resolutions. For each $H$ there exists a first quadrant spectral sequence

$$
E_{p, q}^{2}(H)=\pi_{p} \pi_{V+W+q}^{H}\left(X_{\bullet} \wedge Y_{\bullet}\right)
$$

converging to $\pi_{V+W+p+q}^{H}(X \wedge Y)$.

Proof. In each simplicial dimension, $X_{\bullet} \wedge Y_{\bullet}$ is $(V+W+1)^{*}$-connected, so for each subgroup $H$, the mapping space $\operatorname{Map}_{*}^{H}\left(S^{V+W}, X_{\bullet} \wedge Y_{\bullet}\right)$ is 1-connected $([9$, 4.1]). The spectral sequence of [2], adapted to simplicial spaces by [5] and applied to $\operatorname{Map}_{*}^{H}\left(S^{V+W}, X_{\bullet} \wedge Y_{\bullet}\right)$ converges to $\left|M a p_{*}^{H}\left(S^{V+W}, X_{\bullet} \wedge Y_{\bullet}\right)\right|$. Using an inductive argument on the $G$-CW decomposition of $S^{V+W}$, it can be shown that $\left|\operatorname{Map}_{*}^{H}\left(S^{V+W}, X_{\bullet} \wedge Y_{\bullet}\right)\right|$ is homotopy equivalent to $\operatorname{Map}_{*}^{H}\left(S^{V+W},\left|X_{\bullet} \wedge Y_{\bullet}\right|\right)$ (see $[9,4.3])$. Thus, the $E^{2}$ term is given by $E_{p, q}^{2}(H)=\pi_{p} \pi_{V+W+q}^{H}\left(X_{\bullet} \wedge Y_{\bullet}\right)$ and the spectral sequence converges to $\pi_{V+W+p+q}^{H}\left|X_{\bullet} \wedge Y_{\bullet}\right|$. Now apply Lemma 2.2.

Corollary 2.4. Let $X$ be a based $(V+m-1)^{*}$-connected $G$-CW complex, $Y$ a based $(W+n-1)^{*}$-connected $G$-CW complex, $m, n>0$. For each $H \subseteq G$, there exists an exact sequence

$$
\begin{aligned}
\pi_{V+W+m+n+2}^{H}(X \wedge Y) & \rightarrow E_{2, m+n}^{2}(H) \rightarrow E_{0, m+n+1}^{2}(H) \\
& \rightarrow \pi_{V+W+m+n+1}^{H}(X \wedge Y) \rightarrow E_{1, m+n}^{2} \rightarrow 0
\end{aligned}
$$

Proof. We use the generalized $G(V)$ - and $G(W)$-sphere resolutions of $X$ and $Y$, modified so as to contain only spheres $S^{V+k}, k \geq m$, or $S^{W+\ell}, \ell \geq n$. The simplicial space $X_{\bullet} \wedge Y_{\bullet}$ is $(V+W+m+n-1)$-connected and retains the property that $\left|X_{\bullet} \wedge Y_{\bullet}\right| \simeq_{G} X \wedge Y$. Thus, $E_{p, i}^{2}(H)=\pi_{p} \pi_{V+W+i}^{H}\left(X_{\bullet} \wedge Y_{\bullet}\right)=*$ for $i<m+n, p>0$ and $H \subseteq G$. We have a short exact sequence

$$
0 \rightarrow E_{2, m+n}^{\infty}(H) \rightarrow E_{2, m+n}^{2}(H) \rightarrow E_{0, m+n+1}^{2}(H) \rightarrow E_{0, m+n+1}^{\infty}(H) \rightarrow 0
$$

which, in light of the filtration, yields the exact sequence of the corollary.

\section{UNDERSTANDING THE $E^{2}$ TERM}

In this section we show that the $E^{2}$ terms of the spectral sequences of Theorem 2.3 , when taken as a collection, carry the algebraic structure of a $\Pi(V+W)$-algebra. We will often call this collection of terms the $E^{2}$ term, and this collection of spectral sequences the spectral sequence. We begin by recalling some facts about equivariant П-algebras.

Definition 3.1. Fix any representation $V$ of $G$. Let $\Pi(V)$ be the category with objects $\bigvee_{i=1}^{k} S_{H_{i}}^{V+m_{i}}, m_{i} \geq 1, H_{i} \subseteq G$. The point space $*$ is included as an object of $\Pi(V)$. The morphisms of $\Pi(V)$ are taken to be $G$-homotopy classes of $G$-maps between the objects. A $\Pi(V)$-algebra is a contravariant functor $A: \Pi(V) \rightarrow$ Sets $_{*}$ such that the inclusion maps $i_{j}: U_{j} \hookrightarrow U_{0} \vee U_{1}, j=0,1$, induce an isomorphism $A\left(U_{0} \vee U_{1}\right) \stackrel{\cong}{\rightrightarrows} A\left(U_{0}\right) \times A\left(U_{1}\right)$.

The $\Pi(V)$-algebras we are most interested in are those of the form $[-, X]_{G}$ for $X$ a $G$-space. Such a $\Pi(V)$-algebra is denoted $\Pi_{V}(X)$, and can be thought of as the collection of groups $\left\{\pi_{V+m}^{H}(X)\right\}_{m, H}, H \subseteq G, m \geq 1$, together with the 
operations between these groups. The value of the functor on objects is indeed in the category Gps of groups if $m=1$ and in abelian groups, $A b$, if $m>1$; however, the morphisms are not necessarily group homomorphisms. $\Pi(V)$-algebras form a category with (small) colimits, called $\Pi(V)$-al.

Let $S_{G}$ be the category of subgroups of $G$. By $g r S_{G^{-}}$Sets $s_{*}$ we denote the category of based sets indexed over both positive integers and subgroups of $G$. There is a forgetful functor $\mathcal{U}$ from $\Pi(V)$-al to $g r S_{G^{-}} S e t s_{*}$ with a left adjoint, $\mathcal{F}$. If $T:=\left\{T_{m, H}\right\} \in g r S_{G^{-}} S e t s_{*}$, then the free $\Pi(V)$-algebra $\mathcal{F} T$ is the $\Pi(V)$-algebra associated to a wedge of spheres - one sphere $S_{H}^{V+m}$ for each non-basepoint element $\mu \in T_{m, H}$-ranging over all the sets $T_{m, H}$ in $T$. Thus, the free $\Pi(V)$-algebras are those of the form $[-, U]$, where $U$ is a colimit of objects in $\Pi(V)$. See [19] and [9] for more details.

A simplicial object $A_{\bullet}$ over $\Pi(V)$-al can be thought of as a contravariant functor from $\Pi(V)$ into the category of based simplicial graded sets with the same property as above - that it takes finite wedges to products isomorphically. Applying $\pi_{p}$ to $A_{\bullet}([16, \S 3.6, \S 16.7])$ for $p \geq 0$ produces a $\Pi(V)$-algebra.

Motivated by the $E^{2}$ term of the spectral sequence in Theorem 2.3, we now define the tensor product of a free $\Pi(V)$ - and a free $\Pi(W)$-algebra. We then extend the definition to arbitrary $\Pi(V)$ - and $\Pi(W)$-algebras.

Definition 3.2. Let $A$ and $B$ be free $\Pi(V)$ - and $\Pi(W)$-algebras respectively. Thus $A=[-, X]_{G}$ and $B=[-, Y]_{G}$ for some spaces $X$ and $Y$ which are wedges of equivariant spheres. The tensor product, $A \otimes B$, is the free $\Pi(V+W)$-algebra defined as

$$
A \otimes B=[-, X]_{G} \otimes[-, Y]_{G}:=[-, X \wedge Y]_{G} .
$$

If $A$ and $B$ are arbitrary, choose free simplicial resolutions $A_{\bullet}$ and $B_{\bullet}$ of $A$ and $B$. Since $A_{i}$ and $B_{i}$ are free $\Pi(V)$-, $\Pi(W)$-algebras for each simplicial dimension $i$, $A_{i} \otimes B_{i}$ is defined. With $\otimes$ performed dimensionwise, $A_{\bullet} \otimes B_{\bullet}$ is thus a simplicial $\Pi(V+W)$-algebra. We now define $A \otimes B:=\pi_{0}(A \bullet \otimes B \bullet)$.

It can be shown, in a manner analogous to [19], that $\otimes$ is a well-defined functor. In addition (see [19], [1]), the $p^{\text {th }}$ derived functor, $\otimes_{p}$, of the tensor product functor is well-defined for all $p \geq 0$ by $A \otimes_{p} B:=\pi_{p}\left(A_{\bullet} \otimes B_{\bullet}\right)$ where once again $A$. and $B$. are free simplicial resolutions of $A$ and $B$. By definition, the zeroth derived functor is just the tensor product functor.

With this definition and Theorem 2.3, the first main theorem is now easily deduced.

Proof of Theorem 1.1. For each $H$, we established a spectral sequence converging to $\pi_{V+W+q}^{H}(X \wedge Y), q \geq 1$, with $E_{p, q}^{2}(H)=\pi_{p} \pi_{V+W+q}^{H}\left(X_{\bullet} \wedge Y_{\bullet}\right)$ in Theorem 2.3. For each fixed value of $p, p \geq 0$, the collection of columns $\left\{E_{p, *}^{2}(H)\right\}_{H}$ forms a $\Pi(V+W)$ algebra, namely, $\pi_{p} \Pi_{V+W}\left(X_{\bullet} \wedge Y_{\bullet}\right)$. For each simplicial index $i, \Pi_{V} X_{i}$ and $\Pi_{W} Y_{i}$ are free $\Pi(V)$-, $\Pi(W)$-algebras respectively, so $\Pi_{V} X_{i} \otimes \Pi_{W} Y_{i}=\Pi_{V+W} X_{i} \wedge Y_{i}$. Thus,

$$
\Pi_{V}\left(X_{\bullet}\right) \otimes \Pi_{W}\left(Y_{\bullet}\right)=\Pi_{V+W}\left(X_{\bullet} \wedge Y_{\bullet}\right)
$$

where the indexing is again understood dimensionwise. Since $\Pi_{V}\left(X_{\bullet}\right)$ and $\Pi_{W}\left(Y_{\bullet}\right)$ are free simplicial resolutions of $\Pi_{V}(X)$ and $\Pi_{W}(Y)$, the definition of derived functors now allows us to write for each $p \geq 0$ :

$$
\pi_{p} \Pi_{V+W}\left(X_{\bullet} \wedge Y_{\bullet}\right)=\Pi_{V} X \otimes_{p} \Pi_{W} Y .
$$




\section{4. $\otimes$ AS A KAN EXTENSION}

In Section 4 we took a constructive approach, defining $\otimes$ in order to complete the proof of Theorem 1.1. Using this approach, it is not clear that this functor has any natural properties. We can indeed establish these properties following [19]. However, in this section we will show that $\otimes$ is a left Kan extension (and hence a left adjoint). The advantage of this perspective is that it parallels the definition of the stable box product given by Lewis [11]. We give Lewis's definition here.

Recall that Mackey functors are contravariant, additive functors from the Burnside category $\mathcal{B}$ into abelian groups. The objects of the Burnside category of a group $G$ are finite $G$-sets, and its morphisms are sets of stable homotopy classes of $G$-maps between the associated suspension spectra. This is isomorphic to a full subcategory of the equivariant stable category $([14, \S \mathrm{V} .9])$. (In other words, the objects of $\mathcal{B}$ are often thought of as suspension spectra rather than as finite $G$-sets.)

The box product of two Mackey functors $M$ and $N$ is the left Kan extension of $M \otimes N$ along $\times$, as in the diagram

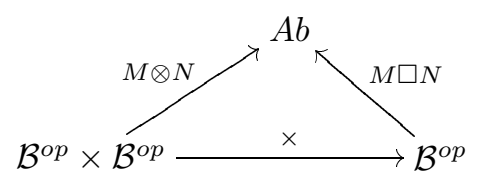

Here $(M \otimes N)(a, b)=M(a) \otimes N(b)$ and $a \times b$ is the product of $a$ and $b$ as finite $G$-sets.

Of course, unstably there is not enough structure to ensure that $\Pi(V)$-algebras take morphisms in $\Pi(V)$ to group homomorphisms. For this reason, unstably we work with the category of sets rather than the category of abelian groups. We also continue to work in the pointed case.

Definition 4.1. Let $A$ and $B$ be $\Pi(V)$ - and $\Pi(W)$-algebras respectively. The unstable box product $A \square B$ of $A$ and $B$ is defined as the left Kan extension of $A \wedge B$ along the functor $\wedge$. Pictorially,

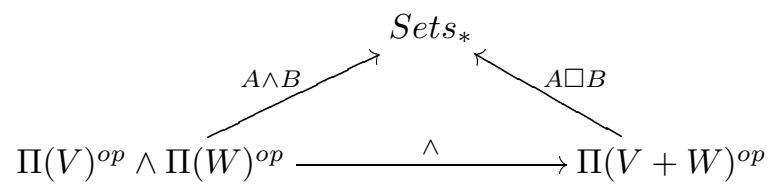

The functor $A \wedge B$ in the diagram is defined on $(c, d)$ as $A(c) \wedge B(d)$. The functor $\wedge: \Pi(V)^{o p} \wedge \Pi(W)^{o p} \rightarrow \Pi(V+W)^{o p}$ is defined on spheres as:

$$
S_{H}^{V+i} \wedge S_{J}^{W+j}=S^{V+W+i+j} \wedge(G / H \times G / J)_{+}=\bigvee_{\ell} S^{V+W+i+j} \wedge\left(G / K_{\ell}\right)_{+}
$$

for some subgroups $K_{\ell}$. For arbitrary objects $a$ and $b$ of $\Pi(V)^{o p} \wedge \Pi(W)^{o p}$, we extend this definition as

$$
a \wedge b=\left(\bigvee_{i} S_{H_{i}}^{V+i}\right) \wedge\left(\bigvee_{j} S_{J_{j}}^{W+j}\right) \cong \bigvee_{i, j}\left(S_{H_{i}}^{V+i} \wedge S_{J_{j}}^{W+j}\right)
$$

Lemma 4.2. Let $\mathcal{C}, \mathcal{D}$ be pointed categories. If $s: \mathcal{C} \rightarrow \mathcal{D}$ is a pointed functor, then the left Kan extension of the representable functor $\mathcal{C}(-, c)$ along $s$ is the representable functor $\mathcal{D}(-s(c))$ for any $c \in \mathcal{C}$. 
Proof. See [10, Chapter 4]; or [15, $\S \mathrm{X}]$ (for the unpointed version).

Lemma 4.3. $A \square B$ is $a \Pi(V+W)$-algebra.

Proof. We need only to show that $A \square B$ maps coproducts to products. When $A=[-, X]_{G}$ and $B=[-, Y]_{G}$ are representable $\Pi(V)-, \Pi(W)$-algebras, Lemma 4.2 gives us that $A \square B=[-, X \wedge Y]_{G}$. This functor clearly maps coproducts to products. When $A$ and $B$ are free $\Pi(V)$-, $\Pi(W)$-algebras, $A=[-, X]_{G}$, and $B=[-, Y]_{G}$, where $X$ and $Y$ are colimits of elements $T_{i} \in \Pi(V), S_{j} \in \Pi(W)$. Namely,

$$
\begin{aligned}
& X=\operatorname{colim}\left(T_{0} \hookrightarrow T_{1} \hookrightarrow T_{2} \hookrightarrow \ldots\right), \\
& Y=\operatorname{colim}\left(S_{0} \hookrightarrow S_{1} \hookrightarrow S_{2} \hookrightarrow \ldots\right) .
\end{aligned}
$$

All the maps are inclusions of wedge summands, and therefore induce inclusions $\left[-, T_{i}\right]_{G} \hookrightarrow\left[-, T_{i+1}\right]_{G}$, and $\left[-, S_{j}\right]_{G} \hookrightarrow\left[-, S_{j+1}\right]_{G}$. The compactness of spheres gives $[-, X]_{G}=\operatorname{colim}_{i}\left[-, T_{i}\right]_{G}$ and $[-, Y]_{G}=\operatorname{colim}_{j}\left[-, S_{j}\right]_{G}$, and we have

$$
\begin{aligned}
{[-, X]_{G} \square[-, Y]_{G} } & =\operatorname{colim}_{i}\left[-, T_{i}\right]_{G} \square \operatorname{colim}_{j}\left[-, S_{j}\right]_{G} \\
& \cong \operatorname{colim}_{i, j}\left(\left[-, T_{i}\right]_{G} \square\left[-, S_{j}\right]_{G}\right) \\
& \cong \operatorname{colim}_{i, j}\left(\left[-,\left(T_{i} \wedge S_{j}\right)\right]_{G}\right) \\
& \cong\left[-, \operatorname{colim}_{i, j}\left(T_{i} \wedge S_{j}\right)\right]_{G} .
\end{aligned}
$$

This functor clearly maps coproducts to products. It is not hard to see that coequalizers preserve this property of mapping coproducts to products.

Lemma 4.4. The functors $-\square D$ and $C \square$ - preserve colimits.

Proof. If $D$ is an arbitrary $\Pi(W)$-algebra, $-\square D$ preserves colimits since it is left adjoint to the generalized Hom functor

$$
\begin{aligned}
\underline{\operatorname{Hom}}_{\Pi(W)}(D,-): \Pi(V+W)-a l & \rightarrow \Pi(V)-a l, \\
F & \mapsto \operatorname{Hom}_{\Pi(W)}(D, F) .
\end{aligned}
$$

Notice that $\underline{\operatorname{Hom}}_{\Pi(W)}(D, F)$ is in fact a $\Pi(V)$-algebra since $F$ maps wedges to products, and since $\underline{\text { Hom }}$ commutes with products in the second variable.

To see that $-\square D$ is a left adjoint to $\underline{\mathrm{Hom}}$, consider that $\operatorname{Nat}_{\Pi(V+W)}(C \square D, F)$ and $\operatorname{Nat}_{\Pi(V) \wedge \Pi(W)}(C \wedge D, F)$ are isomorhphic via the properties of left Kan extensions. Then notice that

$$
\operatorname{Nat}_{\Pi(V) \wedge \Pi(W)}(C \wedge D, F) \cong \operatorname{Nat}_{\Pi(V)}\left(C, \underline{\operatorname{Hom}}_{\Pi(W)}(D, F)\right)
$$

via the usual isomorphism: $(\gamma: C \wedge D \rightarrow F) \mapsto\left(\xi: C \rightarrow \underline{\operatorname{Hom}}_{\Pi(W)}(D, F)\right)$ where $\xi(c)=\gamma(c \wedge-)$.

Let $t w:(\Pi(V), \Pi(W)) \rightarrow(\Pi(W), \Pi(V))$ be the twist map, $t w(a, b)=(b, a)$. Since the diagram

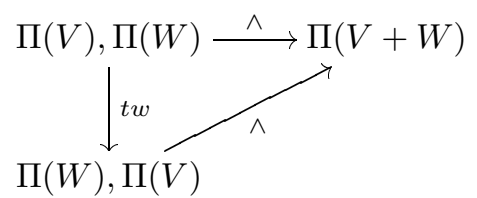

commutes, and since $-\square D$ preserves colimits, $C \square-$ also preserves colimits. 
Lemma 4.5. The functors $-\otimes D$ and $C \otimes-$ preserve sequential colimits.

Proof. Let $\operatorname{colim}_{i} T_{i}$ be a sequential colimit of $\Pi(V)$-algebras $T_{i}$. We first remark that $\operatorname{colim}_{i}\left(T_{i}\right)$ satisfies the definition of free simplicial resolution for the space $\left(\operatorname{colim}_{i} T_{i}\right)$. Thus,

$$
\begin{aligned}
\left(\operatorname{colim}_{i} T_{i}\right) \otimes D & =\pi_{0}\left[-,\left(\operatorname{colim}_{i} T_{i}\right) \wedge D_{\bullet}\right]_{G} \\
& =\pi_{0}\left[-,\left(\operatorname{colim}_{i}\left(T_{i}\right)\right) \wedge D_{\bullet}\right]_{G} \\
& \cong \pi_{0}\left[-, \operatorname{colim}_{i}\left(T_{i} \wedge D_{\bullet}\right)\right]_{G} \\
& \cong \pi_{0} \operatorname{colim}_{i}\left[-, T_{i} \wedge D_{\bullet}\right]_{G} \\
& \cong \operatorname{colim}_{i} \pi_{0}\left[-, T_{i} \wedge D_{\bullet}\right]_{G} \\
& =\operatorname{colim}\left(T_{i} \otimes D\right)
\end{aligned}
$$

As before, commutativity of the twist map $t w$ with $\wedge$ shows that $C \otimes-$ also commutes with sequential colimits.

Remark. In fact, it is a consequence of the next theorem that $-\otimes-$ commutes with all colimits.

Since $A \square B$ is the left Kan extension of $\wedge$ along $\wedge$, there is a one-to-one correspondence between maps from $A \wedge B \rightarrow C \circ \wedge$ and maps from $A \square B \rightarrow C$ for any $\Pi(V+W)$-algebra $C$. Choosing $C=A \otimes B$, notice that when $A$ and $B$ are free $\Pi(V)$-, $\Pi(W)$-algebras, then there is an obvious map $\wedge: A \wedge B \rightarrow A \otimes B \circ \wedge$. If $A$ and $B$ are arbitrary $\Pi(V)$-, $\Pi(W)$-algebras, we take the free $\Pi(V)$-, $\Pi(W)$-algebras $\mathcal{F U} A, \mathcal{F U} B$, on the underlying graded $S_{G}$ sets, and form $A \wedge B$ as the coequalizer

$$
\mathcal{F U}(\mathcal{F U} A) \wedge \mathcal{F U}(\mathcal{F U} B) \stackrel{f_{\mathcal{F U} A} \wedge f_{\mathcal{F U} B}}{\mathcal{F} \underline{\mathcal{U}_{f_{A}} \wedge \mathcal{F U} \mathcal{U}_{f_{B}}}} \quad \mathcal{F U} A \wedge \mathcal{F U} B \longrightarrow A \wedge B
$$

where $f$ is the counit of the $(\mathcal{F}, \mathcal{U})$-adjunction. Similarly, we form $A \otimes B \circ \wedge$ as the coequalizer in the corresponding diagram. In this way $\wedge$ induces a map $A \wedge B \rightarrow A \otimes B \circ \wedge$, which corresponds to a map $\phi: A \square B \rightarrow A \otimes B$.

Theorem 4.6. The map $\phi: A \square B \rightarrow A \otimes B$ is an isomorphism of $\Pi(V+W)$ algebras.

Proof. We begin by assuming that $A$ and $B$ are representable $\Pi(V)$-, $\Pi(W)$-algebras: $A=[-, X]_{G}$ for some $X$ in $\Pi(V), B=[-, Y]_{G}$ for some $Y$ in $\Pi(W)$. Then $[-, X]_{G} \wedge[-, Y]_{G}$ is a representable functor from $\Pi(V)^{o p} \wedge \Pi(W)^{o p}$. Lemma 4.2 now implies that $[-, X]_{G} \square[-, Y]_{G}=[-, X \wedge Y]_{G}$, and this functor is exactly $[-, X]_{G} \otimes[-, Y]_{G}$.

If $A$ and $B$ are free $\Pi(V)$-, $\Pi(W)$-algebras, we can write $A=[-, X]_{G}$ and $B=[-, Y]_{G}$ with $X$ and $Y$ given as sequential colimits as in the proof of Lemma 4.3: $X=\operatorname{colim}\left(T_{0} \hookrightarrow T_{1} \hookrightarrow T_{2} \hookrightarrow \ldots\right)$ and $Y=\operatorname{colim}\left(S_{0} \hookrightarrow S_{1} \hookrightarrow S_{2} \hookrightarrow \ldots\right)$, $T_{i} \in \Pi(V)$ and $S_{j} \in \Pi(W)$. By Lemmas 4.4 and 4.5 , both $\square$ and $\otimes$ preserve sequential colimits, so $\phi: A \square B \rightarrow A \otimes B$ is again an isomorphism when $A$ and $B$ are both free. 
Now, assume that $B$ is an arbitrary $\Pi(W)$-algebra. $\mathcal{F U} B$ is the free $\Pi(W)$ algebra on the underlying graded $S_{G}$ set, and we consider the coequalizer diagrams:

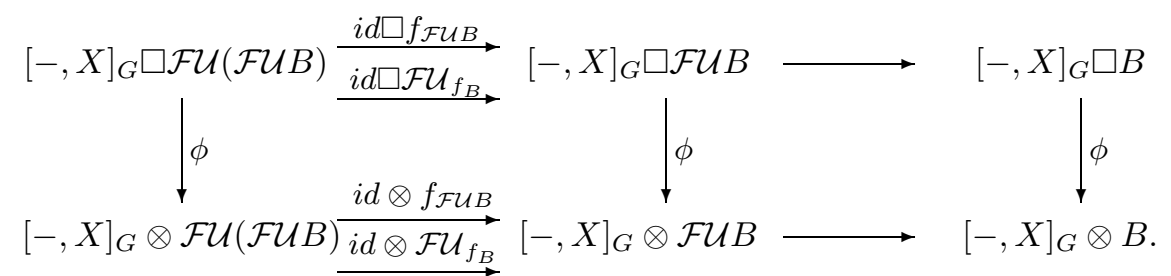

We have already shown that the map $\phi$ is an isomorphism in the two leftmost instances. Thus, by the uniqueness of coequalizers, $\phi:[-, X]_{G} \square B \rightarrow[-, X]_{G} \otimes B$ is also an isomorphism.

We remark that $\phi:[-, X]_{G} \square B \rightarrow[-, X]_{G} \otimes B$ is natural in $X$. This is clear when $B$ is free. When $B$ is an arbitrary $\Pi(W)$-algebra, we again rely on writing $B$ as the coequalizer in

$$
\mathcal{F U F U B} \stackrel{\rightarrow}{F U} B \rightarrow B
$$

Finally, we now apply the natural transformation $\phi:-\square B \rightarrow-\otimes B$ to the coequalizer diagram for an arbitrary $\Pi(V)$-algebra $A$. Since this is an isomorphism on $\mathcal{F} \mathcal{U}(\mathcal{F U} A)$ and $\mathcal{F U} A$, it is also an isomorphism on $A$.

In $\S 3$ the tensor product functor was defined to nicely describe the $E^{2}$ term of the spectral sequence of Theorem 1.1. With the isomorphic description of this functor provided by Theorem 4.6, we can now see that this is a good definition in that it lets us recover some known results from the spectral sequence.

Corollary 4.7. Let $G$ be the trivial group. Let $X$ and $Y$ be based $C W$ complexes such that $X$ is $(m-1)$-connected, $Y$ is $(n-1)$-connected. If $m, n \geq 2$, then $\pi_{m} X \otimes \pi_{n} Y \cong \pi_{m+n}(X \wedge Y)$ as abelian groups.

Proof. Since $X$ is $(m-1)$-connected, the spaces $X_{i}$ in the free simplicial resolution can all be chosen to be $(m-1)$-connected. Likewise, each space $Y_{i}$ in $Y_{\bullet}$ can be chosen to be $(n-1)$-connected. With $V$ and $W$ both taken to be points, the $E_{p, q}^{2}$ term of the spectral sequence of Theorem 1.1 is $\pi_{p} \pi_{q}\left(X_{\bullet} \wedge Y_{\bullet}\right)$. When $q \leq m+n-1$, this is trivial, so $E_{0, m+n}^{2} \cong E_{0, m+n}^{\infty}$. That is,

$$
\left(\Pi_{*}(X) \otimes \Pi_{*}(Y)\right)\left(S^{m+n}\right) \cong \pi_{m+n}(X \wedge Y) .
$$

We will proceed by showing that $E_{0, m+n}^{2}$ is actually $\pi_{m}(X) \otimes \pi_{n}(Y)$ by showing that it has the universal property of the tensor product of abelian groups.

By Theorem 4.6, $\left(\Pi_{*} X \otimes \Pi_{*} Y\right)$ is a left Kan extension, and by [15, $\left.\mathrm{X} \S 4\right]$ it has the coend description:

$$
\left(\Pi_{*} X \otimes \Pi_{*} Y\right)\left(S^{m+n}\right)=\int^{a, b}\left[S^{m+n}, a \wedge b\right] \bullet[a, X] \wedge[b, Y]
$$

where $a, b$ are elements of the category $\Pi(*)$ and $\bullet$ denotes the copower in the category of $\operatorname{Set}_{*}$. We abbreviate this coend by $L\left(S^{m+n}\right)$. Notice that because of the connectivity of $X$ and $Y$, we can assume that $a$ is some finite wedge of spheres of dimension $m$, and $b$ is some finite wedge of spheres of dimension $n$. 
When $a=S^{m}, b=S^{n},\left[S^{m+n}, a \wedge b\right] \bullet[a, X] \wedge[b, Y]$ is a wedge of copies of $\pi_{m} X \wedge \pi_{n} Y$ indexed on the elements of $\left[S^{m+n}, S^{m} \wedge S^{n}\right]$. We then include $\pi_{m} X \wedge \pi_{n} Y$ into this by indexing it on the generator of $\left[S^{m+n}, S^{m} \wedge S^{n}\right]$, which includes into the wedge $\bigvee_{a, b}\left[S^{m+n}, a \wedge b\right] \bullet[a, X] \wedge[b, Y]$ over all elements $a, b$ in $\Pi(*)$. There is a natural, bilinear map $\eta \circ \wedge: \pi_{m} X \times \pi_{n} Y \rightarrow L\left(S^{m+n}\right)$ where $\eta$ is this inclusion followed by the natural map of the summands onto the coend. Now let $\Phi$ be any bilinear map from $\pi_{m} X \times \pi_{n} Y$ into any abelian group $G$. Notice that since $\Phi$ is bilinear, it factors through $\pi_{m} X \wedge \pi_{n} Y$. Call this map $\Phi^{\prime}: \pi_{m} X \wedge \pi_{n} Y \rightarrow G$. We have:

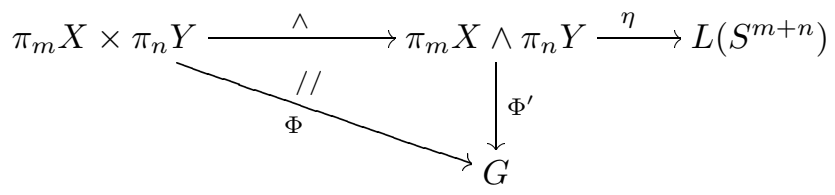

It remains to show that there is a unique homomorphism $\omega: L\left(S^{m+n}\right) \rightarrow G$ such that $\Phi=\omega \circ \eta \circ \wedge$. We will use the universal property of the left Kan extension $L$ to produce this map.

Consider the $\Pi(*)$-algebra which is associated to the Eilenberg-Mac Lane space $K(G, m+n)$. Precomposing this functor with $\wedge$ gives a functor from $\Pi(*) \wedge \Pi(*)$ into $\operatorname{Set}_{*}$. Now define a natural transformation

$$
\alpha:[-, X] \wedge[-, Y] \rightarrow \Pi_{*} K(G, m+n) \circ \wedge
$$

as the trivial map $\left[S^{i}, X\right] \wedge\left[S^{j}, Y\right] \rightarrow\left[S^{i} \wedge S^{j}, K(G, m+n)\right]$ for all pairs $\left(S^{i}, S^{j}\right)$ except $\left(S^{m}, S^{n}\right)$. Define $\alpha_{m, n}:\left(\left[S^{m}, X\right] \wedge\left[S^{n}, Y\right]\right) \rightarrow\left[S^{m} \wedge S^{n}, K(G, m+n)\right]$ to be the map $\Phi^{\prime}$. Now the natural transformation $\omega: L \rightarrow \Pi_{*} K(G, m+n)$ satisfying $\alpha=\omega \circ \eta \circ \wedge$ is produced by the universal property. When restricted to the object $S^{m+n}, \alpha=\Phi^{\prime}$. Thus $\omega$ restricted to $S^{m+n}$ is the desired map.

That $\omega: L\left(S^{m+n}\right) \rightarrow\left[S^{m+n}, K(G, m+n)\right]$ is a group homomorphism and not just a map of sets is a result of the fact that the group structures on $L\left(S^{m+n}\right)$ and on $\left[S^{m+n}, K(G, m+n)\right]$ are derived from the pinch map $S^{m+n} \rightarrow S^{m+n} \vee S^{m+n}$, the fold map $S^{m+n} \vee S^{m+n} \rightarrow S^{m+n}$, and the degree -1 map $S^{m+n} \rightarrow S^{m+n}$. These maps all determine morphisms in $\Pi(*)$. Since $\omega$ is a natural transformation, it commutes with the maps, and hence, with the group structure.

Before we show that the equivariant Freudenthal suspension theorem of [12] also appears along the edge of the spectral sequence of Theorem 1.1, we remind the reader of some terminology.

The $G$-V-Burnside category, $\mathcal{B}_{G} V$, of a group $G$ has as its objects finite disjoint unions of $G$-orbits (or, finite $G$-sets). These are the same objects as in the Burnside category. The morphisms between two objects $c$ and $d$, however, are elements of $\left[\Sigma^{V} c_{+}, \Sigma^{V} d_{+}\right]_{G}$. A $G-V$-Mackey functor is a contravariant functor from $\mathcal{B}_{G}(V)$ into Set $_{*}$ with the property that it maps coproducts to products. In fact, such a functor out of $\mathcal{B}_{G}(V)$ must map into the category of abelian groups. This is the usual description of a $G$ - $V$-Mackey functor: a contravariant additive functor from $\mathcal{B}_{G}(V)$ into $A b$. It is the first description, however, which more closely parallels the case of $\Pi(V)$-algebras. The $G$ - $V$-Mackey functor associated to the based $G$-space $X$ is denoted $\underline{\pi}_{V} X$. 
We remark here that if we fix an integer $m \geq 1$ and consider the subcategory of $\Pi(V)$ with objects of the form $\bigvee_{i=1}^{k} S_{H_{i}}^{V+m}$, there is a canonical bijection between $\mathcal{B}_{G}(V+m)$ and this category. Hence, the restriction of a $\Pi(V)$-algebra to this subcategory is a $G-(V+m)$-Mackey functor. For representations $R \subset R^{\prime}$, there is a functor $s_{*}$ from the category of $G$-R-Mackey functors to the category of $G$ - $R^{\prime}$ Mackey functors. In $[12, \S 5]$, for a $G$-R-Mackey functor $Y, s_{*} \underline{\pi}_{R} Y$ is given as

$$
\left(s_{*} \underline{\pi}_{R} Y\right)(G / K) \cong \int^{d} \mathcal{B}_{G}\left(R^{\prime}\right)(G / K, d) \wedge\left[\Sigma^{R} d, Y\right]_{G}
$$

where $d$ is in the category $\mathcal{B}_{G}(R)$. (See also $[13, \S 4.8]$ for comments on $s_{*}$.)

Corollary 4.8 ([12, Theorem 2.5$])$. Let $Y$ be a based, $(W+n-1)^{*}$-connected $G$ $C W$ complex. If $m \geq 1$, and $n \geq 2$, then

$$
s_{*} \underline{\pi}_{W+n} Y \cong \underline{\pi}_{V+W+m+n}\left(\Sigma^{V+m} Y\right) .
$$

Proof. We first show that $\left(\Pi_{V} S^{V+m} \otimes \Pi_{W} Y\right)\left(S_{K}^{V+W+m+n}\right)$ is isomorphic to the coend description of $s_{*}$ applied to the functor $\underline{\pi}_{W+n} Y$. Then we show that the $E_{0, m+n}^{2}$ term is isomorphic to $E_{0, m+n}^{\infty}$.

By Theorem 4.6, $\Pi_{V} S^{V+m} \otimes \Pi_{W} Y$ is a left Kan extension, and by [15], its value at $S_{K}^{V+W+m+n}$ can be computed by the coend

$$
\int^{a, b}\left[S_{K}^{V+W+m+n}, a \wedge b\right]_{G} \bullet\left[a, S^{V+m}\right]_{G} \wedge[b, Y]_{G}
$$

where $a \in \Pi(V), b \in \Pi(W)$, and $\bullet$ denotes the copower in the category of Sets $_{*}$. The connectivity of $S^{V+m}$ and $Y$, however, means that we can assume that $a$ and $b$ are finite wedges $\bigvee_{i} S_{H_{i}}^{V+m}$ and $\bigvee_{k} S_{J_{k}}^{W+n}$ respectively, where the subgroups $H$ and $J$ can vary, but the dimensions $V+m$ and $W+n$ of the spheres cannot. Thus, $a$ corresponds to an element $c$ in $\mathcal{B}_{G}(V+m)$, and $b$ to an element $d$ in $\mathcal{B}_{G}(W+n)$. We rewrite $\left(\Pi_{V} S^{V+m} \otimes \Pi_{W} Y\right)\left(S_{K}^{V+W+m+n}\right)$ as

$$
\int^{c, d} \mathcal{B}_{G}(V+W+m+n)(G / K, c \times d) \bullet \mathcal{B}_{G}(V+m)(c, G / G) \wedge\left[\Sigma^{W+n} d, Y\right]_{G} .
$$

For fixed $d \in \mathcal{B}_{G}(W+n)$, define $\Psi: \mathcal{B}_{G}(V+m) \rightarrow \mathcal{B}_{G}(V+W+m+n)$ by $\Psi(c)=c \times d$. Now the left Kan extension $L$ of the functor $\mathcal{B}_{G}(V+m)(-, G / G)$ along the functor $\Psi$ is given by the coend

$$
L(G / K)=\int^{c} \mathcal{B}_{G}(V+W+m+n)(G / K, c \times d) \bullet \mathcal{B}_{G}(V+m)(c, G / G) .
$$

Since $\mathcal{B}_{G}(V+m)(-, G / G)$ is a representable functor, by Lemma $4.2, L(G / K)$ can also be expressed as $\mathcal{B}_{G}(V+W+m+n)(G / K, G / G \times d)$. But $G / G \times d \cong d$, so we have

$$
\begin{array}{r}
\int^{c} \mathcal{B}_{G}(V+W+m+n)(G / K, c \times d) \bullet \mathcal{B}_{G}(V+m)(c, G / G) \\
\cong \mathcal{B}_{G}(V+W+m+n)(G / K, d) .
\end{array}
$$

Since $\wedge$ preserves coends, we have 


$$
\begin{aligned}
& \int^{c, d} \mathcal{B}_{G}(V+W+m+n)(G / K, c \times d) \bullet \mathcal{B}_{G}(V+m)(c, G / G) \wedge\left[\Sigma^{W+n} d, Y\right]_{G} \\
\cong & \int^{d}\left(\int^{c} \mathcal{B}_{G}(V+W+m+n)(G / K, c \times d) \bullet \mathcal{B}_{G}(V+m)(c, G / G)\right) \wedge\left[\Sigma^{W+n} d, Y\right]_{G} \\
\cong & \int^{d} \mathcal{B}_{G}(V+W+m+n)(G / K, d) \wedge\left[\Sigma^{W+n} d, Y\right]_{G} \cong\left(s_{*} \underline{\pi}_{W+n} Y\right)(G / K) .
\end{aligned}
$$

This can now be extended from $G / K$ to arbitrary elements of $\mathcal{B}_{G}(V+W+m+n)$.

Finally, since $S^{V+m}$ is $(V+m-1)^{*}$-connected, and $Y$ is $(W+n-1)^{*}$-connected, $E_{p, q}^{2}(K)=*$ for all $K$, when $p \geq 0$, and $q<m+n$. Thus, $E_{0, m+n}^{2}(K)$ is isomorphic to $E_{0, m+n}^{\infty}(K)$ for all $K$. In other words,

$$
\left(\Pi_{V} S^{V+m} \otimes \Pi_{W} Y\right)\left(S_{K}^{V+W+m+n}\right) \cong \pi_{V+W+m+n}^{K}\left(\Sigma^{V+m} Y\right) .
$$

Now notice that since any $G$-map $G / H \rightarrow G / K$ commutes with the differentials of the spectral sequence, Theorem 1.1 actually provides a spectral sequence of $\Pi(V+W)$-algebras. Thus, we have an isomorphism of $G-(V+W+m+n)$-Mackey functors.

\section{REFERENCES}

1. M. André, Méthode simpliciale en algèbre homologique et algèbre commutative, Lecture Notes in Mathematics, 32, Springer, Berlin (1967). MR 35:5493

2. A.K. Bousfield and E.M. Friedlander, Homotopy theory of $\Gamma$-spaces, spectra, and bisimplicial sets, Geometric Applications of Homotopy Theory II, Lecture Notes in Mathematics, 658, Springer, Berlin (1978) 80-130. MR 80e:55021

3. A.K. Bousfield and D.M. Kan, Homotopy Limits, Completions, and Localizations, Lecture Notes in Mathematics, 304, Springer, New York (1972). MR 51:1825

4. G.E. Bredon, Equivariant Cohomology Theories, Lecture Notes in Mathematics, 34, SpringerVerlag, Berlin (1967). MR 35:4914

5. R. Brown and J.L. Loday, van Kampen theorems for diagrams of spaces, with an appendix by M. Zisman, Topology, 26 (1987) 311-335. MR 88m:55008

6. T. tom Dieck, Transformation Groups, Walter de Gruyter, Berlin, 1987. MR 89c:57048

7. W.G. Dwyer, D.M. Kan, and C.R. Stover, An $E^{2}$ model category structure for pointed simplicial spaces, J. Pure and Applied Algebra, 90 (1993) 137-152. MR 95c:55027

8. P.J. Huber, Homotopy theory in general categories, Math. Annalen, 144 (1961) 361-385. MR 27:187

9. M. Intermont, An equivariant van Kampen spectral sequence, Topology and its Applications, 79 (1997) 31-48. MR 98e:55013

10. G.M. Kelly, Basic Concepts of Enriched Category Theory, London Mathematical Society Lecture Note Series 64, Cambridge University Press, Cambridge, 1982. MR 84e:18001

11. L.G. Lewis, Jr, The Box Product of Mackey Functors, unpublished notes.

12. L.G. Lewis, Jr, Equivariant Eilenberg-MacLane spaces and the equivariant Seifert-van Kampen and suspension theorems, Topology and its Applications, 48 (1992) 25-61. MR 93i:55016

13. L.G. Lewis, Jr, The Equivariant Hurewicz Map, Trans. Amer. Math. Soc., 329 (1992) 433-472. MR 92j:55024

14. L.G. Lewis, Jr, J.P. May, M. Steinberger, with contributions by J.E. McClure, Equivariant Stable Homotopy Theory, Lecture Notes in Mathematics, 1213, Springer, Berlin (1986). MR 88e: 55002

15. S. Mac Lane, Categories for the Working Mathematician, Graduate Texts in Mathematics, 5, Springer, New York, 1971. MR 50:7275

16. J.P. May, Simplicial Objects in Algebraic Topology, Van Nostrand, Princeton, 1967. MR 36:5942

17. G. Segal, Categories and cohomology theories, Topology, 13 (1974) 293-312. MR 50:5782 
18. G. Segal, Classifying spaces and spectral sequences, Inst. H. Et. Sci. Math., 34 (1968) 105-112. MR 38:718

19. C.R. Stover, A van Kampen spectral sequence for higher homotopy groups, Topology, 29 (1990) 9-26. MR 91h:55011

20. D.G. Quillen, Spectral sequences of a double semi-simplicial group, Topology, 5 (1966) 155157. MR 33:3302

21. D.G. Quillen, Homotopical Algebra, Lecture Notes in Mathematics 43, Springer, Berlin (1967). MR 36:6480

22. D.G. Quillen, On the (co-)homology of commutative rings, Proc. Symp. Pure Math. XVII, Amer. Math. Soc., Providence, RI (1970) 65-87. MR 41:1722

23. G.W. Whitehead, Elements of Homotopy Theory, Graduate Texts in Mathematics, 61, Springer, New York, 1978. MR 80b:55001

Department of Mathematics, Kalamazoo College, Kalamazoo, Michigan 49006

E-mail address: intermon@kzoo.edu 